Arbeit in ihrem systematischen und umfassenden Charakter. Der vom Autor entwickelte Regimebegriff erscheint forschungspraktisch tragfähig und ist theoretisch höchst reflektiert. Wer nach einer simplen Anbindung an bestehende Regimetheorien sucht, wird insofern nicht enttäuscht, als Barben einleitend klarmacht, dass er keine „starke Theorie“ anstrebt, sondern einen „leistungsfähigen theoretischen Leitfaden" liefern wolle (S. 14). (Freilich erscheint die Begriffswahl somit nicht mehr zwangsläufig.) Die wichtigste Leistung der Arbeit besteht jedoch in der systematischen Klärung des sowohl wissenschaftlich als auch öffentlich kontrovers diskutierten Verhältnisses von Neoliberalismus und moderner Biotechnologie. Hätte zwar ein Stichwortregister den Zugang zur verarbeiteten Materialfülle erleichtert, sorgen eine klare Gliederung und Texthervorhebungen doch für gute Übersichtlichkeit.

\langle\rangle

\section{Eine Ethik für die Zukunft zur Steuerung der Gegenwart}

\section{A. Grunwald: Auf dem Weg in eine nano- technologische Zukunft. Philosophisch- ethische Fragen. Freiburg i. Br.: Karl Al- ber, 2009, 388 S., ISBN 978-3-495-48327-5, $€ 22,00$}

\section{Rezension von André Gazsó, Institut für Technikfolgen-Abschätzung, Wien}

Was können wir von den Nanotechnologien erwarten und wie sollen wir mit diesen Erwartungen in der Gegenwart umgehen? Armin Grunwalds Buch zu philosophisch-ethischen Fragen der Nanotechnologien ist eine systematische Auseinandersetzung mit den Hauptargumenten der gegenwärtigen Debatte um die Nanotechnologien, deren Forschungsperspektiven und möglichen Anwendungen. Und als solche kommt sie gerade zur rechten Zeit, denn wenn sich auch die derzeit noch zaghafte öffentliche Diskussion um die Nanotechnologien hauptsächlich um Vermutungen, Möglichkeiten und Spekulationen dreht, so scheint sie sich v. a. in Europa in letzter Zeit zunehmend auf gegenwartsrelevante Bereiche (Regulierung von Nanomaterialien, Arbeits- und Konsumentenschutz, Gestaltung aktueller und zukünftiger Forschungspolitik) zu beziehen. Grunwald leistet mit seinem Buch einen wichtigen Beitrag zur Orientierung in der Diskussion über einen sinnvollen gesellschaftlichen Umgang mit Ungewissheit im Zusammenhang mit neuen und schwer $\mathrm{zu}$ fassenden - und damit schwer vermittelbaren - Technologien. Indem er am Beispiel der Nanotechnologien alle Schattierungen des Wirklichkeitsbezugs in seine Erörterung aufnimmt (von den sehr konkreten Anforderungen im Umgang mit Nanopartikeln bis hin zu den eher spekulativen Fragen im Zusammenhang mit der technischen Verbesserung des Menschen) gelingt ihm diese Bezugnahme überzeugend.

\section{Einführung - Ethik der Nanotechnologie}

Die Zielsetzung des Buches ist klar: Sie besteht darin, ,aktuelle und absehbare Entwicklungen in der Nanotechnologie mit den dabei involvierten Zukunftsvorstellungen für Mensch und Gesellschaft systematisch in philosophischer und ethischer Hinsicht zu untersuchen" (S.12). Es wird dabei jedoch weder der Versuch unternommen, eine eigene Bereichsethik - etwa analog der Bioethik - zu schaffen, noch wird der Begriff der Ethik auf eine allgemeine Betrachtung gesellschaftlicher Aspekte der Nanotechnologie ausgeweitet. Grunwald bemüht sich ganz im Gegenteil darum, die Verfahren der philosophischen Ethik auf die Nanotechnologien anzuwenden. Das ist insofern wertvoll, als in dieser Arbeit deutlich wird, dass Ethik als philosophische Fachdisziplin, ja die Philosophie überhaupt, sehr wohl etwas zu aktuellen Problemen zu sagen hat, gerade dort, wo es darum geht, normative Unsicherheiten zu reflektieren. Orientierungsprobleme, die sich zu normativen Konflikten entwickeln können, ergeben sich im Zuge technischer Innovationen nicht selten (solche mit hohem individuellem ,Spaßpotenzial" einmal ausgenommen) und sind im Falle der Nanotechnologien insofern prekär, als sich diese bisher aus vielerlei Gründen (hohe Interdisziplinarität, Pluralität denkbarer Anwendungen und Entwicklungsstadien, Fehlen einer verbind- 
lichen Definition) jedem Abgrenzungsversuch widersetzten. Grunwald zufolge kann und soll ethische Reflexion hier durch „konditional-normative Beratung einen Beitrag zur Bewältigung dieser Unsicherheiten leisten“( S. 13).

\section{Ethische Fragen der Nanotechnologien}

Im ersten der drei Teile des Buches (Kapitel 2-4) wird zunächst eine fundierte Einführung in die Nanotechnologien geboten, wobei v. a. der Darstellung der Definitionsproblematik und der Darstellung der momentanen gesellschaftlichen Debatte ausreichend Platz gewidmet wird. Darauf folgen, noch als Teil der Grundlegung, ein sehr wertvolles Kapitel zur Problemorientierung der Ethik, in dem Kriterien der Ethikrelevanz dargelegt werden, und ein Kapitel zum Verhältnis von Ethik und Technik.

Der zweite Buchteil (Kapitel 5-9) beschäftigt sich mit substanziellen Fragen der Nanotechnologien und ihrer Anwendungen. Einleitend wird zunächst erörtert, wie die ethische Reflexion der Nanotechnologien überhaupt in das Feld der Ethik einzuordnen ist. Grunwald plädiert hier dafür, keine eigene „Nano-Ethik“ als eigene Teildisziplin zu begründen - sie wäre dann nichts anderes als eine weitere „Bindestrich-Ethik“, sondern die ethische Reflexion der Nanotechnologien als „begriffliche und konzeptionelle Plattform“ (S. 116) aufzufassen, ,auf der ethische Deliberation, interdisziplinärer Dialog und demokratische Auseinandersetzung" stattfinden können (S. 16). Über die Facetten dieser ethischen Auseinandersetzung legt er dann im darauf folgenden Kapitel einen systematischen Überblick vor. Es handelt sich in erster Linie um Fragen der Vorsorge, der Verteilungsgerechtigkeit, des menschlichen Kontrollanspruchs, der medizinischen Nutzungsmöglichkeiten und möglicher bzw. befürchteter Grenzüberschreitungen zwischen Technik und menschlicher Natur auf Basis eines technischen Machbarkeitsanspruchs.

Als konkrete Anwendungsbereiche werden in diesem zweiten Teil der Einsatz synthetischer Nanopartikel, die Möglichkeiten der Nanobiotechnologie und die „Verbesserung“ des Menschen mit Mitteln der Nanotechnologien ausführlich behandelt. V. a. der Einsatz von synthetischen Nanopartikeln in der Produktion von Konsumartikeln wirft eine Fülle an regulierungsrelevanten Fragen auf und steht momentan im Mittelpunkt des öffentlichen und politischen Interesses, da sich in dieser Größendimension bei gleicher chemischer Zusammensetzung neuartige Eigenschaften ergeben. Es waren ja gerade diese neuen Eigenschaften, die ursprünglich den Proponenten der Nanotechnologie so viel versprechend erschienen, dass sie - zunächst in den USA, dann in Europa und Asien - die Etablierung großer nationaler Forschungsprogramme und Aktionspläne rechtfertigen sollten. Umso erstaunlicher ist es dann, wenn man sich, quasi zur Abwehr öffentlicher Befürchtungen über mögliche Schäden, wieder auf bestehende Forschungstraditionen beruft. Dieses argumentatorische Vor-und-Zurück ist allerdings bereits aus vorangegangenen Technologiewahldebatten bekannt. Wenn auch die entsprechende toxikologische, toxikokinetische und zellbiologische Forschung zur Wirkung von synthetischen Nanopartikeln seit einigen Jahren auf nationaler und internationaler Ebene zunehmend gefördert wird (die EU gab für diesen Bereich im 6. Rahmenprogramm etwa $30 \mathrm{Mrd}$. Euro aus, in den ersten drei Jahren des 7. Rahmenprogramms sind es bereits ebensoviel), so sind die bisherigen Erkenntnisse aus dieser Forschung noch spärlich und sehr divers. Gleichzeitig verstärkt sich in den letzten Jahren die öffentliche Forderung nach Regulierung der Anwendung von synthetischen Nanopartikeln in bestimmten Sektoren (v. a. verbrauchernahe Produkte), was insbesondere den Druck auf jene Behörden erhöht, die für Arbeitnehmer- und Konsumentenschutz zuständig sind.

Die Frage nach einem adäquaten Umgang mit Unwissenheit, also der angemessenen Risikovorsorge ohne ausreichende wissenschaftliche Grundlage, verlange nach einer konkreten Ausdeutung des Vorsorgeprinzips, so Grunwald, um hier handlungsfähig zu bleiben. Dieses für Interessenten des Risikomangements sehr wichtige 7. Kapitel identifiziert die entsprechenden normativen Streitfragen im Zusammenhang mit etwaigen Vorsorgenotwendigkeiten und schlägt eine mögliche Strategie rationaler Konfliktbewältigung vor.

Die beiden folgenden Kapitel befassen sich mit der möglichen Überschreitung der Grenzen zwischen dem Technischen und dem Lebendigen: 
Kapitel 8 erörtert ethisch relevante Fragestellungen einer synthetischen Biologie, also der Anwendung ingenieurwissenschaftlicher Prinzipien in biologischen Systemen. Dies erscheint auf Nanometer-Maßstab durchaus machbar, wenn sich auch hier - gleich wie in den Nanotechnologien - sehr Vieles noch im Bereich der Grundlagenforschung abspielt. Kapitel 9 widmet sich der ethischen Debatte um die „technische Verbesserung“ des Menschen. Auch hier sollen Nanotechnologien entscheidende Beiträge leisten, etwa bei der Entwicklung von Neuroimplantaten. Problematisiert werden speziell Ansätze, die über etablierte therapeutische Eingriffe hinausgehen. Dabei müsse zunächst einmal die Frage geklärt werden, was nun eigentlich unter „Verbesserung“ zu verstehen sei, um in diesem Zusammenhang überhaupt ethische Fragen präzise formulieren zu können (S. 250 ff.).

\section{Mögliche Zukünfte}

Wie Grunwald eingangs in seinem Buch betont, befasst sich die ethische Reflexion der Nanotechnologien überwiegend mit den Hoffnungen und Befürchtungen, die diese in der gesellschaftlichen Diskussion initiieren und freisetzen. Wir haben es also, anders als bei vorangegangen öffentlichen Technikdebatten, hauptsächlich mit der Diskussion um vielfältige Zukunftsentwürfe zu tun. Das allerdings rückt sie wieder näher an den grundlegenden Ansatz klassischer prospektiver Risikobewertung, wo es ebenfalls um eine Abwägung möglicher Konsequenzen gegenwärtiger Entscheidungen geht, aber eben mit dem Unterschied, dass im Falle der Nanotechnologien diese Abwägung noch weitgehend ohne Hilfe entsprechender Daten historischer Schadensfälle auskommen muss. Es gehe also in der Debatte vornehmlich um ,die Zukünfte, die sie [die Nanotechnologie], metaphorisch gesprochen, verspricht oder mit denen sie droht", so Grunwald (S. 12).

Und eben dieser Erfassung teils weit reichender Zukunftsaussagen in der ethischen Debatte und der damit verbundenen Schwierigkeiten im Umgang mit dem hohen Grad an Unsicherheit ist der letzte Teil des Buches gewidmet. Prospektive Ethik wirft nämlich eine Reihe epistemologischer Fragen auf, da wir bereits bei der Bestimmung normativer Unsicherheiten auf mehr oder weniger unsicheres Folgenwissen angewiesen sind. Das betrifft nun nicht nur die mangelnde Bestimmbarkeit der Eintrittswahrscheinlichkeit möglicher Ereignisse (deren Bestimmung ist bereits in der klassischen Risikoabschätzung mit erheblichen Problemen behaftet), sondern auch der Höhe möglicher Schäden, ja der Schadensart überhaupt. Grunwald unternimmt also im letzten Teil dankenswerter Weise den Versuch, die „Abhängigkeit der Ethik von prospektiven Annahmen und ihre Angewiesenheit auf epistemologische Reflexion dieser Annahmen" systematisch nachzugehen (S. 315). Zunächst einmal geht es überhaupt um die Geltung prospektiver Aussagen, über die nach dem Muster aktueller Aussagen diskursiv entschieden wird, wobei betont werden muss, dass auch über prospektive Aussagen nur auf der Basis bereits bestehender Wissensbestände und nach Kriterien der jeweiligen Gegenwart geurteilt werden kann. Das nimmt den Erwartungen, den positiven (Verheißungen) wie den negativen (Apokalypsen), zwar etwas von ihrer (beabsichtigen) appellativen Spitze, befreit sie jedoch auch von ihrem Joch der Beliebigkeit. Grunwald zeigt an dieser Stelle sehr schön, dass auch aus der Ungewissheit noch ein Maß an Bestimmtheit gewonnen werden kann, indem er eine Abstufung von Wissens- und Nichtwissensbeständen vornimmt: gegenwärtiges Wissen (und dieses wird dann wieder wissenschaftlich validiert - eine wertvolle Arbeit, die im aktuellen Fall der Nanotoxikologie von wissenschaftlichen Gremien wie dem Scientific Committee on Emerging and Newly Identified Health Risks (SCENIHR) unternommen wird), Einschätzungen zukünftiger Entwicklungen, ceteris-paribus Bedingungen und Ad-hoc-Annahmen. Das führt die beliebige Spekulation wieder auf das Feld „begründeter Erwartbarkeiten“, im Falle der Risikoabschätzung vom dystopischen worst case zum plausible worst case. Den Rest des epistemologischen Kapitels ist der systematischen Behandlung prospektiver ethischer Aussagen gewidmet, v. a. der Entfaltung brauchbarer Beurteilungsverfahren (Assessments) möglicher Zukünfte, wobei zwischen einem ethisch orientierten Foresight im Falle kurzfristiger Ansätze und eines Vision Assessment für weitreichende Entwürfe unterschieden wird. 
Insgesamt also ist Armin Grunwalds Buch $\mathrm{zu}$ philosophisch-ethischen Fragen der Nanotechnologie ein wertvoller Beitrag zur Aufarbeitung der momentan noch etwas orientierungsund differenzierungslosen öffentlichen Debatte in diesem Bereich, da es nicht nur eine lang erwartete (und beinahe schmerzlich vermisste) Systematisierung der ethischen Auseinandersetzung mit diesen Technologien unternimmt, und v. a. im letzten Teil neue Erkenntnisse und Lösungsansätze anbietet. Empfohlen sei es für alle jene, die sich einen Überblick über den momentanen Stand der öffentlichen Debatte über die Nanotechnologie verschaffen wollen, aber auch für jene, die in mitten tatsächlicher Handlungsanforderungen nach Orientierung ringen. Um es mit einem, von dem österreichischen Schriftsteller Friedrich Torberg kolportierten Ausspruch zu sagen: „Mit Genuss und Belehrung gelesen“.

$\langle\langle\rangle$

\section{Der technisch erweiterte Mensch}

What does it mean to be human?

\section{Zonneveld, H. Dijstelbloem, D. Ringoir (eds.): Reshaping the Human Condition. Exploring Human Enhancement. The Hague: Rathenau Institute (in collabora- tion with the British Embassy, Science and Innovation Network and the Parlia- mentary Office of Science and Technolo- gy) 2008,176 S., ISBN 978-90-77364-24-6}

\section{Rezension von Leonhard Hennen, ITAS}

„How far should things be taken?“ Diese Frage, die Kevin Warwick, einer der Autoren des vorliegenden Sammelbandes und Professor für $\mathrm{Cy}$ bernetics (University of Reading, UK) sich auch im Hinblick auf seine eigene experimentelle Forschung zu Mensch-Maschine-Schnittstellen stellt, kann als Leitfrage des hier rezensierten Buches wie auch der gesamten Diskussion über „Human Enhancement" gelten. Das Thema „Human En- hancement", d. h. die gezielte technische Erweiterung oder Steigerung menschlicher Fähigkeiten über das „Normale“ hinaus, ist eine relativ junges Thema wissenschafts- und technologiepolitischer Debatten, hat aber bereits zu einer Reihe von $\mathrm{Pu}$ blikationen auch aus dem Bereich der Technikfolgenabschätzung geführt (z. B. STOA 2009; Merkel et al. 2007; vgl. auch die laufende Studie des TAB „Pharmakologische und technische Interventionen zur Leistungssteigerung"). Die Karriere dieses Themas ist eng verknüpft mit der technologiepolitischen Diskussion um die Konvergenz der neuesten Entwicklungen in den Nano-, Informations- und Kommunikations- sowie Neurowissenschaften. Damit - so einige Propagandisten der "Converging Technologies“ - würde ein neuer Schub technischer Entwicklung ausgelöst, der (u. a. durch neue Möglichkeiten der Verkoppelung von Organischem und Technischem) Wege einer künstlichen Erweiterung menschlicher Leistungsfähigkeit eröffnen würde (vgl. Coenen 2008).

Der vom niederländischen Rathenau-Institut herausgegebene Sammelband will sowohl die aktuellen und zukünftigen technischen Möglichkeiten des Enhancements als auch die sozialen und ethischen Implikationen dieser Entwicklung ausloten. Die versammelten Beiträge gliedern sich in einen ersten Teil, der sich mit den ,ethics and impacts", und einen zweiten, der sich mit „applications and expectations“ "befasst. Ergänzt werden diese Beiträge durch zwei Interviews zu grundsätzlichen Fragen der Mach- und Wünschbarkeit einer Erweiterung der kognitiven Leistungsfähigkeit. Der Band geht auf eine Tagung zurück, die vom herausgebenden Rathenau Institut zusammen mit der britischen Botschaft und dem Parliamentary Office of Science and Technology des britischen Parlamentes im Jahr 2007 durchgeführt wurde.

Wie in der Enhancement-Debatte generell, stehen die Erweiterung der menschlichen kognitiven Fähigkeiten und damit die Neurowissenschaften samt pharmakologischen sowie informationstechnischen Möglichkeiten der „Verbesserung" menschlicher mentaler Leistungen im Zentrum der Beiträge. Typische Beispiele, die in den Beiträgen diskutieret werden, sind etwa die Verbesserung von Aufmerksamkeit und Wachheit oder des Erinnerungsvermögens durch Psy- 